\title{
Acciones necesarias para mejorar la relación causa-efecto entre la inversión en prácticas de gestión de recursos humanos y la motivación en la empresa
}

\author{
José-Luis Rodríguez-Sánchez \\ Dpto. Economía de la Empresa (ADO), Economía Aplicada Il y Fundamentos del Análisis Económico, Universidad Rey \\ Juan Carlos, Madrid - España. (correo-e: joseluis.rodriguez.sanchez@urjc.es)
}

Recibido Ago. 20, 2019; Aceptado Oct. 17, 2019; Versión final Nov. 7, 2019, Publicado Abr. 2020

\begin{abstract}
Resumen
Se propone un modelo para gestionar la motivación de recursos humanos integrando las aportaciones de los autores especialistas en el tema. Para ello, se llevó a cabo un caso de estudio en una empresa líder del sector de la ingeniería mecánica durante cinco años. La empresa está presente en los cinco continentes, en más de 100 países y tiene más de 60.000 empleados. Con los resultados del caso de estudio, las empresas pueden implantar medidas para gestionar la motivación de los trabajadores, gestionando los factores fundamentales de éxito detectados. Se demuestra que una plantilla motivada contribuye a la creación de sinergias, al crecimiento personal de los empleados y a un mejor posicionamiento de la empresa como buena empleadora. Si los trabajadores están motivados, ayudan a atraer trabajadores valiosos del mercado y se retiene a los trabajadores con talento de la empresa.
\end{abstract}

Palabras clave: motivación; gestión de recursos humanos; cultura; clima laboral; satisfacción.

\section{Necessary actions to improve the cause-effect relationship between investment in human resources management practices and motivation in the company}

\begin{abstract}
A model for managing human resources motivation, incorporating the observations of specialized people in the field is proposed. For this, a study was developed during five years in a leading enterprise of the mechanical engineering area. The company has business in all five continents, with more than 60,000 employees, and is active in more than 100 countries. With the results of the case studied other companies can adopt a number of measures aimed at improving the management of staff's motivation, enhancing the key success factors in the workplace. It is demonstrated that motivated workers contribute to the creation of synergies, workers' personal development, and ultimately to a better reputation of the company as a good employer. Succeeding in motivating your staff can attract highly-rated workers in the market to your organization and it can even serve as a powerful tool to retain the better valued, talented employees.
\end{abstract}

Keywords: motivation; human resources management; culture; working atmosphere; satisfaction. 


\section{INTRODUCCIÓN}

En el entorno empresarial actual los directores y líderes de las empresas deben encontrar y gestionar prácticas para conseguir una alta eficiencia de los recursos humanos (en adelante, $\mathrm{RH}$ ) si quieren llegar a ser competitivos. La competitividad en las organizaciones esta explicada por factores del entorno, en general fuera de su control, y por factores internos relacionados con su gestión. Los factores internos tienen mayor incidencia y explican por qué en una misma industria -sometida a los mismos factores del entorno-, hay empresas que obtienen mejores resultados que otras (Miles, et al., 2018). Pero en muchas ocasiones a pesar de implantar y gestionar prácticas de gestión de $\mathrm{RH}$ los resultados de las encuestas de satisfacción indican que existe desmotivación. El problema existente es: ¿por qué empresas que invierten tiempo y recursos en gestión de $\mathrm{RH}$, tienen a sus empleados desmotivados e incluso muchos están buscando otras empresas para trabajar?

La complejidad de la motivación laboral se basa en que requiere que se tengan en cuenta los factores ambientales y personales de los RH (López-Mas, 2005). Las tendencias actuales de gestión obligan a una necesaria congruencia entre los procesos administrativos y psicológicos para la optimización del funcionamiento organizacional (Palma-Carrillo, 2000). Se están desarrollando nuevas culturas empresariales que rompen con las tradicionales prácticas de organización del trabajo. Entre estas transformaciones destaca la apuesta por establecer una gestión participativa de los $\mathrm{RH}$. La participación de los $\mathrm{RH}$ permite, por un lado, aprovechar los conocimientos de los trabajadores y, por otro, garantizar su mayor implicación en la consecución de los objetivos empresariales. De esta manera se consigue el incremento de la motivación y rendimiento de los propios trabajadores (Lahera-Sánchez, 2006).

La gestión empresarial de $\mathrm{RH}$ se entiende, como el proceso de influir en los trabajadores para que contribuya a los objetivos de los individuos y de la organización en conjunto. Para ello se requiere la creación y mantenimiento de un contexto en el que los individuos trabajen juntos hacia el logro de objetivos comunes (López-Mas, 2005). Aspectos del entorno interno empresarial, como el atractivo de los sitios de trabajo, la cultura organizacional y la marca, deben considerarse por su influencia en la innovación de las organizaciones (Bernal-Torres y Blanco-Valbuena, 2017). En la gestión de RH se considera que la motivación y, por extensión, la satisfacción del individuo en su ámbito laboral influye en que el trabajador colabore con los objetivos de la empresa. En consecuencia, la implicación del individuo en su trabajo tiene una importancia estratégica (Arevalo-Tomé et al., 2009). La correcta implantación de medidas encaminadas a mejorar la satisfacción de los empleados tanto a nivel individual como colectivo conlleva obtener una organización más eficaz y que sea difícilmente imitable por otras empresas, ya que las personas que forman parte de la organización son un recurso único de cada empresa y pueden ser la fuente de ventajas competitivas sostenibles.

El trabajo tiene dos objetivos. El objetivo del trabajo para la comunidad científica, se genera un modelo teórico integrado sobre los factores de gestión de $\mathrm{RH}$ determinantes para conseguir una organización motivada en base a las aportaciones previas de los autores especialistas en el ámbito de investigación. En segundo lugar, para la práctica empresarial, con la información obtenida durante cinco años de interacción directa e informal en una empresa líder del sector de la ingeniería mecánica se genera un marco de acciones y buenas prácticas que los líderes y directores pueden realizar para conseguir una exitosa gestión de la motivación en sus organizaciones.

El trabajo se ha estructurado en varias secciones. En primer lugar, se ha realizado una revisión de la literatura existente en el ámbito de la gestión de RH y la motivación. En segundo lugar, se han analizado las diferentes perspectivas teóricas desde los dos puntos de vista que conciernen al objetivo del trabajo, por un lado, la gestión de los $\mathrm{RH}$ y por otro lado se analiza la motivación relacionada con los trabajadores y el entorno laboral. En el siguiente apartado del trabajo, se ha explicado la metodología utilizada y los resultados del caso de estudio en el que se analiza una empresa multinacional líder del sector de la ingeniería mecánica. Se ha creado un modelo que profundiza en los factores más influyentes y la forma en que se pueden gestionar para obtener una organización motivada. Por último, se ha expuesto la discusión del trabajo y las conclusiones.

\section{OTROS ANTECEDENTES}

Se analizan las diferentes perspectivas teóricas desde los dos puntos de vista que conciernen al objetivo del trabajo, por un lado, la gestión de los $\mathrm{RH}$ y por otro lado se analiza la motivación relacionada con los trabajadores y el entorno laboral. Para obtener la literatura previa en el tema de investigación se ha realizado una revisión sistemática de la literatura. Una revisión sistemática de la literatura es un estudio selectivo y crítico que trata de analizar e integrar la información esencial de estudios primarios de investigación sobre un asunto concreto. Este procedimiento identifica estudios relevantes, valorando su calidad y sintetizando sus resultados. La revisión sistemática se considera un estudio secundario, pues su población de estudio la constituyen los propios estudios primarios. Las revisiones deben realizarse de forma objetiva, rigurosa y 
meticulosa (Manterola, 2009). La revisión supone una tarea muy metódica que requiere: 1) identificar los estudios ya existentes, 2) analizar su utilidad y relevancia en un determinado tema de investigación y, 3) reunir un número de estudios realizados de manera independiente, a veces con resultados opuestos, y sintetizar sus implicaciones.

La realización de una revisión sistemática requiere una estrategia de búsqueda de información a partir de bases de datos bibliográficas, una valoración apropiada de los estudios que serán incluidos en el ulterior análisis y, finalmente, una adecuada síntesis de la información recopilada. Esta recopilación de información debe ser exhaustiva para evitar incurrir en un sesgo de selección (no incluir estudios relevantes provoca un error sistemático en la estimación del efecto), para lo cual hay que buscar en el mayor número de fuentes posible, con criterios de selección adecuados. La búsqueda de artículos se llevó a cabo con fecha 26/05/2019 empleando el protocolo de búsqueda que aparece en la Tabla 1.

Tabla 1. Protocolo de búsqueda.

\begin{tabular}{|c|c|c|}
\hline Base de Datos & WoS & Scopus \\
\hline Alcance geográfico & Bibliografía científica mundial & Bibliografía científica mundial \\
\hline Características & $\begin{array}{l}\text { Indicadores de calidad: } \\
\text { Factor de impacto JCR } \\
\text { Índice de inmediatez } \\
\text { Número de citas } \\
\text { Cuartiles }\end{array}$ & $\begin{array}{l}\text { Indicadores de calidad: } \\
\text { Factor de impacto SJR } \\
\text { Número de citas } \\
\text { Cuartiles }\end{array}$ \\
\hline Documentos buscados & Artículos de revista & Artículos de revista \\
\hline Criterios de búsqueda & Título del artículo & Título del artículo \\
\hline Rango de datos & Todos los años hasta 2019 & Todos los años hasta 2019 \\
\hline Fecha de búsqueda & 22 octubre 2019 & 22 octubre 2019 \\
\hline Términos de búsqueda & $\begin{array}{l}\text { "Motivation" } \\
\text { "Human Resources Management" }\end{array}$ & $\begin{array}{l}\text { "Motivation" } \\
\text { "Human Resources Management" }\end{array}$ \\
\hline Proceso de filtrado: Categorías & Management and Business & Business, Management and Accounting \\
\hline
\end{tabular}

Las fuentes que se han utilizado en este trabajo para realizar la búsqueda bibliográfica de artículos de revistas son dos bases de datos: 1) Web of Science (WoS) y 2) Scopus. Son las bases de datos más prestigiosas en el ámbito de las Ciencias Sociales, especialmente en el área de la Economía y Empresa. Es un recurso valioso para los investigadores ya que ofrecen información sobre indicadores de calidad y parámetros como el número de citas o el índice de inmediatez que permiten analizar y clasificar las publicaciones.

\section{Gestión de los Recursos Humanos}

El papel de los directores de RH ha cambiado mucho a lo largo de la historia. El factor humano dentro de la teoría económica clásica era considerado como una parte más del engranaje de la empresa, es decir un simple agente encargado de la transformación de inputs a outputs (Arévalo-Tomé et al., 2009). Posteriormente fue evolucionando y en la década de los noventas la gestión de los $\mathrm{RH}$ cambió de estrategia, se centró en un enfoque interno basado en los recursos, que buscaba la utilización óptima de los activos y habilidades de la empresa como forma de obtener una ventaja competitiva (Chiavenato, 2011).

Las cualidades clave de los $\mathrm{RH}$ hacen referencia a la cultura corporativa, el grado de compromiso con la empresa, las habilidades que poseen o la capacidad de aprendizaje. Dichos recursos intangibles son difícilmente imitables por parte de los competidores y es por este motivo que las estrategias referidas al factor humano se consideran particularmente relevantes a la hora de crear una ventaja competitiva sostenida en el tiempo (Jiang et al., 2012). Además, si reconocemos la complejidad que entraña la gestión de los RH podemos deducir que la empresa precisa de una habilidad propia que le haga posible combinar dichos recursos, integrarlos y movilizarlos a su favor a través de una serie de prácticas organizativas (Grant, 1991). La estrategia de $\mathrm{RH}$ de una empresa quedará definida por dichas decisiones y rutinas organizativas que toma. Para que dicha estrategia tenga éxito no solo debe contar con trabajadores y con los recursos apropiados, sino que en la empresa también debe de tener gestores capaces de sacar el máximo provecho a sus subordinados. Esta mejora no se producirá si no se estimula adecuadamente a los trabajadores, por lo que se precisa cierta psicología organizativa para motivar al trabajador (Guerra y Sansevero, 2008). La gestión de 
$\mathrm{RH}$ es una combinación de habilidades, métodos, políticas, técnicas y prácticas definidas con el objetivo de administrar los comportamientos internos y potenciar el capital humano. Tiene el objetivo de seleccionar, administrar y guiar a los empleados hacia los objetivos y metas de la empresa (Martini et al., 2015).

La administración de $\mathrm{RH}$ significa conquistar y mantener personas en la empresa, trabajando y dando lo mejor de sí, con una actitud positiva y favorable. Los principales objetivos de la administración de $\mathrm{RH}$ son crear, mantener y desarrollar un contingente de personas con habilidades, motivación y satisfacción para alcanzar los objetivos de la empresa. (Chiavenato, 2011). Las prácticas de gestión de $\mathrm{RH}$ son aquellas que aumentan las capacidades y competencias de los empleados para alcanzar los objetivos de la organización (Jiang et al., 2012). La correcta interpretación de los datos e informes sobre los $\mathrm{RH}$ pone de manifiesto la estrecha relación entre la satisfacción y motivación del personal con el aumento de la productividad, con la mejora de la calidad y del servicio en beneficio de los clientes (Álvarez-López, 1995). Por ello los gestores deben de tener sumo cuidado a la hora de interpretar la información que les llega sobre el desempeño del personal a su cargo, ya que es de vital importancia para crear un clima de trabajo óptimo.

\section{La motivación}

La motivación es una característica de la psicología humana que contribuye al grado de compromiso de la persona; es un proceso que ocasiona, activa, orienta dinamiza y mantiene el comportamiento de los individuos hacia objetivos esperados (López-Mas, 2005). El estudio de la motivación para el trabajo es tratar de entender cuáles son las razones o motivos que influyen en el rendimiento de las personas, que son la fuerza impulsora detrás de la producción de bienes y prestación de servicios (Maximiano, 1995).

Existen dos variables relacionadas con la motivación: la competitividad, vista como el deseo de ser mejor que los demás, y la instrumentalidad orientada a la asociación percibida por el trabajador entre su aportación a la empresa y la recompensa recibida (Brown y Peterson, 1993). Los trabajos que se desarrollan basándose en tareas con una alta variedad, autonomía, identidad y feedback pueden generar y reforzar un alto nivel de motivación (Arévalo-Tomé et al., 2009). En el ámbito laboral es importante conocer las causas que estimulan la acción humana, ya que, mediante el manejo de la motivación, entre otros aspectos, los administradores pueden operar estos elementos a fin de que su organización funcione adecuadamente y sus miembros se sientan más satisfechos (Lahera Sánchez, 2006). Para conseguir una mejora continua en la organización y mantener una ventaja competitiva es absolutamente necesario disponer de unos RH adecuadamente motivados (Álvarez-López ,1995).

El éxito de una organización radica en su personal, ya que la empresa se valora por la calidad de sus empleados. Por ello es esencial formarlos, motivarlos e integrarlos en el proyecto empresarial (Álvarez López, 1995). Es muy complicado identificar cuáles son los factores que realmente motivan a los trabajadores tanto de manera individual como colectiva, lo que dificulta vincular los objetivos de los trabajadores hacia los de la empresa (Palma-Carrillo, 2010). La motivación del personal se basa en una serie de principios entre los que cabe destacar: 1) El principio de comunicación establece que si el personal conoce cuales son los factores de los que dependen los resultados a alcanzar, aumentará la motivación que impulsa el logro de los objetivos propuestos (Míguez, 2005). 2) El principio de reconocimiento se basa en que las personas necesitan afecto, sentir que pertenecen a un grupo de referencia, tener conciencia y sobretodo reconocimiento de la tarea bien realizada (Lahera-Sánchez, 2006). 3) Los principios de delegación y participación están relacionados y expresan que gracias a conceder al personal el poder necesario para tomar las decisiones encaminadas para cumplir con los objetivos propuestos, se aumentará la participación y el entusiasmo del personal (ÁlvarezLópez, 1995). Los directores y líderes deben considerar el uso de técnicas como la formación de grupos, la delegación y la autogestión para lograr un alto rendimiento laboral (Guerra y Sansevero, 2008).

Existen teorías que versan sobre la motivación en el entorno laboral se pueden clasificar en dos grandes grupos, primero las teorías de contenido son aquellas que buscan expresar cuales son las causas que estimulan la motivación del individuo, donde podemos englobar la teoría de la motivación humana (Maslow, 1943), la teoría bifactorial (Herzberg, 1966) y la teoría de las necesidades (McClelland, 1965). Y segundo, las teorías de proceso que buscan explicar cuál es el proceso de motivación de una persona, se trata de teorías que proponen soluciones. Se engloban en la teoría de las expectativas (Vroom, 1964), la teoría del refuerzo (Skinner, 1971) y en la teoría de la equidad (Adams, 1963).

\section{METODODOGÍA}

Para dar respuesta a la pregunta de investigación: ¿por qué empresas que invierten tiempo y recursos en gestión de $\mathrm{RH}$, tienen a sus empleados desmotivados e incluso muchos están buscando otras empresas para trabajar? Se propone modelo que integra los factores de gestión de RH determinantes para la motivación y se analiza un caso de estudio de una fusión internacional llevada a cabo por una empresa multinacional. La 
empresa estudiada ha conseguido dominar el sector de la ingeniería mecánica y tener presencia en los cinco continentes a través de desarrollo externo utilizando estrategias de fusión y adquisición. Actualmente, cuenta con más de 60.000 empleados y tiene presencia en más de 100 países. Durante su trayectoria se ha expandido geográficamente, desarrollando nuevos mercados a través de operaciones internacionales de F/A, por lo que es una compañía con suficiente experiencia para considerar los resultados obtenidos válidos y fiables. La Tabla 2 muestra las etapas seguidas en el caso de estudio.

Tabla 2. Etapas del caso de estudio

\begin{tabular}{|l|}
\hline Semblanza \\
Revisión de literatura (WoS, Scopus) \\
\hline Selección de la muestra \\
Compañía líder sector ingeniería mecánica \\
Multinacional opera en más de 100 países \\
Tiene más de 60.000 empleados \\
\hline Selección e identidad de las unidades de análisis \\
Cuatro miembros del comité de dirección \\
Más de 40 informantes de diferentes niveles y departamentos \\
\hline Recolección de información \\
Entrevistas semiestructuradas: 2016 -18 (miembros del comité de dirección) \\
Entrevistas abiertas: 2017-2018 (informantes diferentes niveles y departamentos) \\
Documentos internos: 2013-2018 \\
Observación directa: 2013-2018 \\
(Principio de triangulación y saturación teórica) \\
\hline Transcripción de información \\
ATLAS.ti \\
Registro y clasificación de los datos: \\
1) documentos internos, 2) entrevistas \\
3) notas de campo y 4) observación directa \\
\hline Presentación de resultados y conclusiones \\
Conformidad con los resultados del análisis \\
Conclusiones e implicaciones literarias y profesionales \\
\hline
\end{tabular}

Perry (1998) expone que el caso de estudio, dentro de la investigación cualitativa, ayuda al entendimiento en profundidad de un fenómeno o problema social concreto. Su uso es especialmente relevante cuando el propósito es entender, solucionar o mejorar un procedimiento llevado a cabo en el mundo profesional (Villareal-Larrinaga y Landeta-Rodríguez, 2010). En este trabajo se ha utilizado la metodología del caso de estudio por tres razones: 1) el fenómeno investigado es exploratorio y descriptivo, 2) la literatura no es concluyente y es necesario realizar un análisis en profundidad y cualitativo sobre el tema, y 3) se dispone de información primaria gracias a la colaboración de la empresa estudiada que ha permitido realizar entrevistas a personas involucradas en el proceso y la estancia de uno de los investigadores dentro de la organización.

Se ha realizado una estancia en la empresa de cinco años. Para la recolección de evidencia se han realizado entrevistas con los diferentes informantes con el objetivo de promover un diálogo abierto. Se ha trabajado con dos grupos de personas. Por un lado, cuatro miembros del comité de dirección (director de $\mathrm{RH}$, director de operaciones, director de formación y desarrollo y director de comunicación y marketing). Con los que se han realizado dos entrevistas semiestructuradas con cada uno de ellos. Además, después de la transcripción de la información los resultados han sido revisados por ellos, para verificar que la idea escrita es similar a la idea expresada. Por otro lado, se han realizado 46 entrevistas abiertas y, además se ha establecido contacto informal durante los cinco años con personas de diferentes niveles organizativos y diferentes departamentos. La diversidad de la procedencia de los informantes - tanto por su escalafón organizativo como por el departamento de procedencia - aporta diferentes perspectivas que permiten obtener conclusiones e implicaciones relevantes sobre la investigación. El número de entrevistas e informantes no se concretó al inicio de la investigación y se optó por seguir una estrategia de "saturación teórica" para determinarlo. Se realizaron entrevistas hasta que se percibió que los informantes reportaban información similar y coherente para soportar la validez y calidad del modelo teórico (Yin, 1998). 
Para complementar las entrevistas, se participó en reuniones, planes de comunicación o eventos organizados por la compañía donde se promovían los valores de la organización en materia de gestión de RH como el compromiso y el desarrollo de $\mathrm{RH}$, o la integridad y la confianza. Y para completar el principio de triangulación en la recolección de la información se ha podido acceder a documentos internos. A través de la intranet corporativa se consultaron diferentes documentos, destacando el manual de ejecución de la estrategia de $\mathrm{RH}$ 2013-2018 creado y firmado por el director de RH del grupo a nivel mundial. Dentro del manual destaca el lema "apoyar nuestro negocio mediante el apoyo a nuestros empleados".

\section{MODELO DE GESTIÓN DE FACTORES DE RH}

Existen múltiples factores en la gestión de las personas que pueden servir para generar una plantilla motivada y con sus objetivos alineados con los de la organización, en el trabajo se han destacado factores mostrados en la Figura 1, una vez analizada la literatura sobre el tema de investigación y el estudio en profundidad a través del caso de estudio. El director de RH indicó: "todos los miembros de una organización no tienen las mismas necesidades, ya que todas las personas son diferentes, partiendo de esta base las motivaciones de unos y otros serán a su vez distintas. Algunas personas se sentirán atraídas por los bienes materiales y otras necesitan el reconocimiento a su tarea. Será la misión de los gestores conocer cuáles son las necesidades de sus subordinados, para así poder saber cuál es el mejor camino para incentivar su desempeño".

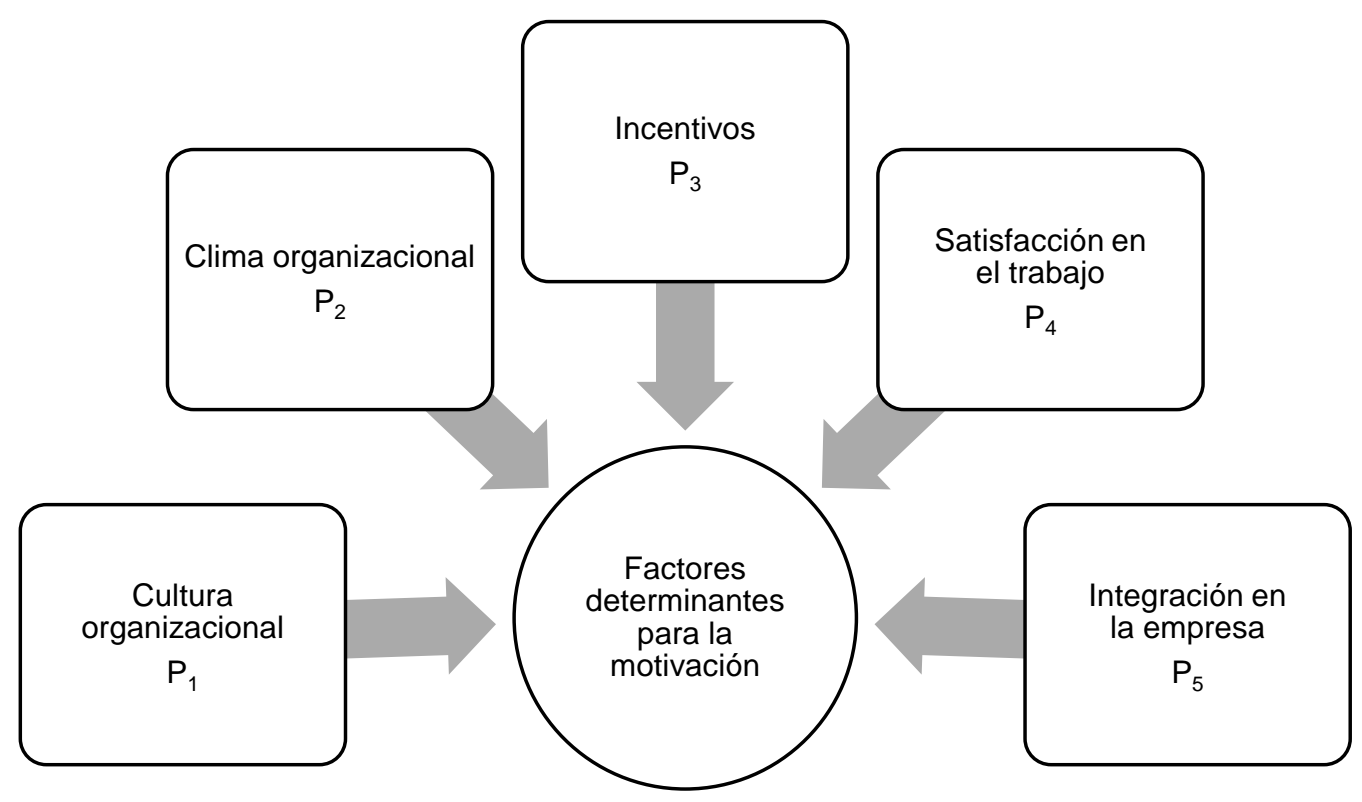

Fig. 1: Factores de gestión de recursos humanos determinantes para la motivación

La motivación es el motor de la conducta humana. El interés por una necesidad pone en funcionamiento un mecanismo que incita a la persona a la acción. Cada vez que aparece una necesidad se rompe el estado de equilibrio del organismo y produce un estado de tensión, insatisfacción e inconformismo que lleva al individuo a desarrollar una acción capaz de descargar la tensión. Una vez satisfecha la necesidad el organismo retorna a su estado de equilibrio (Carrillo et al., 2009). La motivación tiene su origen en ciertos estímulos que actúan con fuerza sobre los individuos, llevándolos a la acción, pudiendo producirse por variables endógenas o exógenas. Esa acción se denomina ciclo motivacional. Este ciclo está formado por las siguientes etapas: equilibrio interno, estímulo, tensión, necesidad, acción y satisfacción.

Cuándo el ciclo motivacional no consigue realizarse, generalmente sucede la frustración del individuo, que mantiene algunas actitudes como: un comportamiento anormal, se puede mostrar agresivo por no conseguir satisfacer algo que deseaba, puede sufrir nerviosismo, con respecto al enfoque de su actitud en el trabajo puede sufrir una falta de interés por las tareas u objetivos, incluso dicha persona puede sentir pasividad, baja moral, pesimismo, inseguridad y resistencia al cambio (Martini et al., 2015). Cuando no se consigue satisfacer alguna necesidad, la persona estará frustrada y ansiosa, como consecuencia de la frustración puede sufrir algunas alteraciones en su comportamiento. Pero no significa que el individuo permanecerá eternamente frustrado. De algún modo la necesidad será transferida o compensada, así percibimos que la motivación es un estado cíclico y constante en la vida personal (Marañón-Rodríguez, et all., 2006). 


\section{La cultura organizacional}

La cultura organizacional es un sistema de valores compartidos por los miembros de una empresa en todos los niveles, que diferencia a una organización de las demás. (Martini et al., 2015). Es un conjunto de prácticas que pueden ser materiales e inmateriales, que dan cuenta de las características que distinguen a una empresa. Establece una atmosfera afectiva común y un marco cognitivo compartido (Cao y Li, 2010). La cultura organizacional desempeña varias funciones, en primer lugar, les da a sus integrantes una identidad organizacional para compartir normas, valores y percepciones. Proporcionando un sentido de unidad para promover un sentimiento de propósito común. Existen diferencias entre las culturas organizacionales fuertes y débiles. Una cultura fuerte sirve para regular el comportamiento de las personas vinculadas con la organización, reforzando el control y la coordinación interna, mejorando la alineación entre las metas de la organización y de las personas (Kotter y Heskett, 1992).

Respecto a la importancia de la cultura organizativa el director de operaciones afirmó: "la cultura de empresa tiene un fuerte valor en la organización y puede ser uno de los vehículos para lograr una diferenciación con respecto a los competidores". Además, el director de $\mathrm{RH}$ añadió sobre la importancia de la cultura en la gestión del talento: "una empresa puede conseguir a través de la cultura organizacional, no solo retener al personal con mayor valor, sino que se puede atraer a excelentes trabajadores de otras organizaciones que deseen formar parte de esa determinada empresa". La cultura facilita el compromiso colectivo, promueve la estabilidad organizacional, y moldea el comportamiento. La comunicación se convierte en un factor determinante para el desarrollo de los grupos, la lucha por el logro de los objetivos comunes, la motivación, la participación y la disciplina, como aspectos sobre los que se debe actuar para alcanzar tal propósito. El director de comunicación y marketing al respecto indicó: "la comunicación interna desde su punto de vista descendente sirve para unir y cohesionar a las personas en torno a un proyecto participativo. Mediante la comunicación descendente la empresa transmitirá no sólo lo que realmente es, sino incluso lo que desea o quiere ser, y ello lo hará a través de los muchos mensajes que se pueden enviar".

Respecto al tipo de cultura el director de administración explicó: "como la mayoría de las grandes organizaciones tenemos una cultura dominante procedente de la familia fundadora, con características influidas por su personalidad y país de origen. Pero el mayor problema de gestión cultural proviene de que el modo de expansión ha sido a través de fusionarse con empresas objetivo. Lo que ha conllevado la aparición de muchas tensiones, resistencias e intentos mantener antiguas subculturas. Estas subculturas se hacen muy evidentes entre diferentes departamentos y países".

Respecto al tipo de cultura el director de RH indicó: "se debe de fomentar la existencia de una cultura fuerte en la empresa y para ello son cruciales que se den prácticas de gestión basadas en la equidad. Desde el departamento de gestión de personas se sepa interpretar cuales son los valores informales de la organización que se deben de fomentar, tenemos identificados cuales son los $\mathrm{RH}$ valiosos que deben ser el modelo ideal para seguir por el resto de los compañeros y mediante el proceso de comunicación trabajamos para convertirlos en ejemplos. Los premios anules a mejor trabajador, las promociones internas o la selección para los programas de formación de talentos son ejemplos de acciones muy bien valoradas por los $\mathrm{RH}$ de la empresa". P1: la satisfacción con la cultura organizativa aumentará la motivación de los RH.

\section{El clima organizacional}

Cada una de las organizaciones cuenta con sus propias características y propiedades a veces únicas y exclusivas. La manera en que se manifiesta el clima organizacional repercute en el comportamiento del individuo trabajador y por consiguiente en la productividad en la empresa (Sandoval-Caraveo, 2004). El clima organizacional es el indicador de satisfacción de los $\mathrm{RH}$ de una empresa, en relación con diferentes aspectos como modelo de gestión, proceso de comunicación, valoración profesional e identificación con la empresa (Marañón-Rodríguez et al., 2006). Gestionar exitosamente los factores de RH tiene un papel fundamental en la capacidad de la organización de promover un buen clima laboral (Tay et al., 2017).

El clima organizacional y la motivación son dos importantes indicadores del funcionamiento psicológico de los $\mathrm{RH}$ en las organizaciones (Salazar-Estrada et al., 2009). Cuando es alta la motivación entre los RH de la organización, el clima organizacional tiende a ser elevado y proporciona relaciones de satisfacción, de animación y de colaboración entre los integrantes de la empresa. Por otro lado, cuando hay baja motivación, el clima organizacional tiende a empeorar. Cuando el clima es bajo se caracteriza por estados de desinterés o apatía entre otros (Chiavenato, 2011). El director de comunicación y marketing explica: "es fundamental que existan una serie de canales de información que permitan chequear, en diversos momentos cual es el ambiente que se respira en los diferentes departamentos de la empresa. El mensaje transmitido debe ayudar a los $\mathrm{RH}$ a sentirse orgullosos de pertenecer a la organización. Para ello funciona muy bien comparar los puntos fuertes de la organización con el resto de las empresas del sector". 
El director de RH añade: "la comunicación interna facilita la obtención de la información necesaria para conocer el clima laboral de la empresa. Implantamos sistemas organizados, ágiles y fluidos de comunicación entre la dirección y el personal, a través de los canales que se establezcan y que hagan posible que las personas puedan sentirse más involucradas en sus actuaciones de cada día. Sin duda, la comunicación interna permite no solo conocer el momento presente, sino también el horizonte de futuro, lo que crea un clima de confianza, motivación e ilusión. Por ejemplo, se utiliza un buzón de sugerencias, encuestas de satisfacción o evaluaciones del desempeño con el objetivo de captar los datos que permitan conocer los posibles motivos de insatisfacción del personal, sus preferencias y deseos de formación, autodesarrollo, promoción y participación. El objetivo es tratar de promover la autorrealización, satisfacción, integración y motivación de los empleados".

El director de formación y desarrollo para fomentar la mejora del clima laboral indicó: "se debe de promover la puesta en práctica de medidas para incentivar los comportamientos que generan un clima organizacional acorde con los valores de la empresa. Y sobretodo, reconocer a los trabajadores con dichas actitudes que queremos promover con recompensas o reconocimiento personal y público en la revista corporativa o en los planes de comunicación. El objetivo es que sean vistos por el resto de los $\mathrm{RH}$ como ejemplos de personas con valores propios de la empresa, consiguiendo que dichas formas de actuar se repitan luego por otros miembros de la organización, siendo estas actitudes vistas como comportamientos deseables". Un clima organizacional propicio incrementará las iniciativas para promover nuevos proyectos, solucionar problemas, mejorará la comunicación interna, aumentará la competitividad y facilitará el gobierno de la organización. Pero para ello requiere la motivación necesaria para dinamizar y garantizar el desarrollo de unas políticas adecuadas por parte de los líderes. $\mathrm{P}_{2}$ : el clima organizacional positivo aumentará la motivación de los RH.

\section{Los incentivos}

Los incentivos tienen el objetivo de estimular o premiar el desempeño, además suponen el reconocimiento. El reconocimiento y la autoestima son necesidades importantes para cualquier persona y pueden satisfacerse a través de los programas de incentivos., competencia interna o proyecto de mejora del rendimiento de las personas y de la empresa (Robbins, 2010). La construcción de un contrato psicológico puede hacer que las dos partes tengan un sentimiento positivo de buenas condiciones, la creación de la atmósfera y el espacio de confianza mutua, la solidaridad, la cooperación, el desarrollo común, inspirando el entusiasmo y la energía de los empleados con el fin de desarrollar su potencial y todo su talento, también para hacer el mejor uso de la eficiencia y la eficacia en la gestión de $\mathrm{RH}$ (Cao y Li, 2010).

Existen diferentes incentivos como los materiales, logro motivacional, el clima interno y la motivación emocional. Según los autores Orlovic et al., (2015) los RH no están satisfechos con su empresa debido a la falta de gestión de factores como la gestión del desempeño, la falta de gestión del desarrollo de carrera y la poca autonomía en sus tareas. La evaluación y gestión del desempeño justa y una comunicación de feedback clara y argumentada por los superiores una vez evaluados, es un factor muy influyente en la motivación de los $\mathrm{RH}$ (Lee, 2019).

Respecto a los incentivos materiales el director de operaciones indicó: "los incentivos materiales representan los incentivos más básicos y fáciles de cumplir porque son las aspiraciones más comunes de los trabajadores. Por ejemplo, salarios, bonos o viajes entre otros. Pero cuando comenzamos a gestionar RH valiosos nos dimos cuenta el dinero es un medio y no un fin. El dinero puede motivar a unos miembros de la organización mientras que a otros no, incluso analizando las diferentes etapas de las carreras profesionales se puede llegar al punto en que los integrantes a los que les había motivado el dinero, ya no les sirva como aliciente en otro momento distinto del tiempo".

El director de RH habló sobre el logro motivacional muy relacionado con los incentivos que buscan los $\mathrm{RH}$ valiosos: "en la actualidad muchos trabajadores eligen trabajar no solo por cubrir sus necesidades más básicas, también buscan obtener una sensación de logro y satisfacción. Este tipo de incentivos son muy importantes en la motivación de los empleados, para ello la empresa debe facilitar la participación de los empleados en el sistema de gestión de la empresa, delegando en ellos mayores responsabilidades y autotomía en la toma de decisiones, fomentar los programas de formación como el mentoring donde tienes un tutor del comité de dirección mundial. Es un programa con un duro proceso de selección, que una vez dentro tiene una duración de seis años y en los cuales se forma a los directivos del futuro".

El director de administración resaltó la importancia de la justicia en la selección y promoción en el clima interno o ambiente de trabajo: "se implantan una serie de políticas y regulaciones que pueden garantizar la equidad del personal, ya que la justicia percibida es un factor importante en las necesidades del personal. Los empleados creen que, si trabajan en una empresa que tiene un ambiente equitativo y justo, habrá menos injusticias en las decisiones de los directores y líderes, por lo tanto, mejorará la eficiencia del trabajo. Se tiene que luchar contra la selección y promoción por compromisos personales ya que es una de las principales fuentes de desmotivación mostrada por el $88 \%$ de los RH en las encuestas de satisfacción". 
La motivación emocional la valoró el director de comunicación y marketing: "se busca la construcción de un ambiente cercano, cordial y armonioso entre los integrantes de la empresa para motivar al personal. Para es fundamental el papel de los seres queridos y familia, por ejemplo, celebración de fiestas de cumpleaños o realización de actividades fuera de las sedes de la empresa con familiares como tardes en el circo o cine". Se debe utilizar una variedad de incentivos de forma combinada para conseguir el objetivo de cambiar el modo de pensar en los trabajadores. Para así establecer un sistema de incentivos que pueda adaptarse a las características de las empresas, a las características de los empleados y a las necesidades de los distintos momentos del tiempo, sobretodo en una etapa como la actual. $\mathrm{P}_{3}$ : otorgar los incentivos adecuados aumentará la motivación de los $\mathrm{RH}$.

\section{La satisfacción en el trabajo}

La implicación del individuo con sus tareas tiene una importancia estratégica para la empresa. La satisfacción de los $\mathrm{RH}$ es uno de los requisitos que contribuye a mejorar el rendimiento de la empresa (Yeung y Berman, 1997). En la gestión de $\mathrm{RH}$ valiosos un trabajo que sea mentalmente desafiador es clave. Este tipo de $\mathrm{RH}$ prefieren trabajar en puestos en los que tengan la oportunidad de usar sus capacidades, habilidades $y$ conocimientos. Un trabajo sin desafíos y monótono les desanima creándoles frustración y un sentimiento de fracaso (Robbins, 2010). El enfoque causa-efecto supone que la calidad de vida de un $\mathrm{RH}$ depende de su satisfacción en muchas áreas concretas como salud, relaciones sociales, familia, finanzas o trabajo (Ortiz et al. 2019). Dentro del proceso de desarrollo continuo y gestión de la carrera de los $\mathrm{RH}$ el director de formación y desarrollo destacó: "los empleados deben ser polivalentes, autoeficientes, autónomos y flexibles, y si están preparados para ello tener un desarrollo de carrera evolutivo y con los plazos de tiempo adecuados. Un $\mathrm{RH}$ valioso si esta más de dos años realizando las mismas tareas entrara en un estado de monotonía y sensación de estancamiento. Se deben ofrecer posibilidades de promoción".

Respecto al trabajo diario, el director de operaciones explicó: "el ambiente, el trato y las condiciones afectan a nuestra voluntad, motivación y al desarrollo de nuestra carrera profesional. Pero tiene especial relevancia la existencia de un exitoso proceso comunicación a través de la cual el empleado sepa claramente donde está y a dónde va. El objetivo, evitar desmotivación debido a la incertidumbre de que pasará en un entorno empresarial tan competitivo y cambiante. Por último, otro aspecto fundamental en la satisfacción de los empleados con su puesto de trabajo es que los horarios de trabajo sean flexibles para disponer de tiempo libre, ya sea para conciliar su vida familiar o personal. Dado el avance tecnológico, el empleo de ordenadores portátiles, teléfonos móviles u tablets hacen poder optimizar el tiempo de trabajo". El director de RH destacó el factor más importante para la satisfacción en el trabajo: "la existencia de compañeros que den apoyo tanto personal como profesional es crucial en la satisfacción laboral. Entre los factores que llevan a evitar la insatisfacción en el trabajo se incluyen las relaciones interpersonales y las condiciones de trabajo. El entorno de la organización se consideran cuatro características indispensables para la motivación: los compañeros de trabajo, las relaciones interpersonales con el superior, las políticas de recompensas y el sistema de promoción".

La satisfacción en el trabajo es un aspecto clave para evitar el principal problema al que se enfrentan las empresas en la actualidad: la dificultad para retener a $\mathrm{RH}$ valiosos. El director de $\mathrm{RH}$ confirmó este problema: "existe relación entre satisfacción y absentismo, según la cual los empleados insatisfechos en sus puestos de trabajo suelen faltar más a su empleo. La satisfacción también se relaciona con la rotación de la plantilla y si existen altos índices de insatisfacción habrá altos índices de rotación en la empresa. Para solucionar el problema del elevado número de abandonos voluntarios realizamos una entrevista a todos los $\mathrm{RH}$ que deciden abandonar la organización. El 87\% indicaban que el estrés generado por la poca relacional con su superior y el $94 \%$ afirmaba que no tenían relacional con los $\mathrm{RH}$ de otros departamentos".

Este es un problema muy grave y el director de operaciones habló de las medidas para solucionar: "la existencia de una alta tasa de rotación supone costes inherentes a formación y capacitación del personal de nuevo ingreso y la correspondiente pérdida de valor humano que se deriva de la marcha de trabajadores valiosos y con potencial hacia la competencia. Pusimos el foco del problema en los diferentes directores y líderes para que fueran los primeros en modificar su actitud. En la última encuesta de satisfacción el $68 \%$ de los $\mathrm{RH}$ considera que ha mejorado la relación con su superior". $\mathrm{P}_{4}$ : la satisfacción con las tareas desempeñadas aumentará la motivación de los $\mathrm{RH}$.

\section{La integración en la empresa}

Los $\mathrm{RH}$ integrados en la empresa se sienten contentos con su trabajo y considera la empresa como una prolongación de su hogar. De esta forma no buscará evadirse sus tareas diarias, sino que buscará su perfeccionamiento. La integración conlleva la mejora de la calidad, la disminución de los costes y el aumento de la productividad (Álvarez-López, 1995). Las empresas en la actualidad buscan establecer una relación lo más fluida posible entre las distintas áreas o departamentos de la empresa, de forma que cada área sienta que forma parte de la organización empresarial, constituyendo entre todos debidamente unidos una gran 
energía optimizando de esta forma los esfuerzos individuales. Los líderes son la línea de unión entre los $\mathrm{RH}$ y las organizaciones. Sus funciones son conseguir la satisfacción mutua de ambas partes y cumplir los objetivos y planes marcados por la organización. La comunicación entre líderes y empleados y es la clave para comprender los deseos y planes de la dirección y las posibilidades de conseguir los resultados esperados con los RH que integran la organización (Dadic et al., 2018).

Con la comunicación horizontal se busca poner fin a los departamentos estancos, con el objetivo de que todos los miembros de la organización remen en la misma dirección y hacer que todos los integrantes puedan sentirse miembros cualificados de una tarea común (Lago y Wilson, 1995). Promover la participación de los miembros de la institución en la organización, difusión y consecución de actividades de reconocimiento institucional son actuaciones necesarias para fortalecer la cultura organizacional basándose en la integración de los miembros de la organización (Palma-Carrillo, 2000). La gestión de los factores de RH relacionados con la integración e involucración en la transferencia de conocimiento, trabajo en equipo y resolución de problemas, no solo ayuda a motivar a los $\mathrm{RH}$, también influye en la atracción y retención de $\mathrm{RH}$ valiosos con alto rendimiento (Rajiani et al., 2016). La atracción, desarrollo y retención de recursos humanos valiosos en las empresas es considerada una fuente de ventaja competitiva sostenible (Rodríguez-Sánchez et al., 2018).

El director de RH habló de la importancia del proceso de socialización: "cuando un nuevo miembro comienza a formar parte de la organización es muy importante el proceso de integración que dicho individuo sufre, cobra especial relevancia en dicho momento la transmisión de la cultura empresarial y que sea acogido de forma que sienta todo el apoyo de la empresa. Gracias a ello los valores de la organización seguirán una realimentación positiva y este trabajador procederá de esta forma similar cuando se incorporé otro nuevo empleado. Utilizamos los llamados budie, determinados $\mathrm{RH}$ valiosos que explican y aconsejan acerca forma de actuar en la empresa, además de que le exponga cuales son los valores y costumbres de la organización que no están escritos".

Además, el director de comunicación y marketing resaltó la implicación del proceso de comunicación: "la comunicación interna se infunde seguridad al individuo que acaba de llegar, gracias a ella los trabajadores se sentirán más integrados al ser miembros de un sistema participativo. Cobra especial relevancia la comunicación transversal como elemento integrador de la empresa con los nuevos trabajadores. Se crea un plan de acogida cuyo objetivo sea facilitar la comunicación de la persona que se incorpora con sus compañeros de trabajo desde los primeros días. En este proceso comunicativo tiene una especial importancia la tarea formativa del jefe jerárquico para la recepción de la persona de nuevo ingreso y su adecuación y adaptación al puesto de trabajo".

En la tarea de integrar a los $\mathrm{RH}$ de la empresa tiene mucha incidencia la forma de actuar de los directores y líderes de los diferentes departamentos. El directo de operaciones al respecto indicó: "la integración es una tarea importantísima de la dirección y por ello cada supervisor debe valorar y respetar a la persona por lo que vale. Los empleados que tiene a sus órdenes son seres humanos que poseen inquietudes, sentimientos y un enorme potencial creativo. Informar y escuchar a sus subordinados es fundamental en la comunicación interna. El objetivo es conocer cada vez mejor a los $\mathrm{RH}$ de la organización, enriqueciéndose con sus puntos de vista e integrándose con ellos en la tarea común que tienen encomendada. Por último, el director de comunicación y marketing: "buscamos tener equipos preparados y capacitados para poder delegar en los colaboradores. Es muy importante para la integración. Necesitamos directores valoran a sus empleados, les informa y los escucha, ya que nadie puede ni debe hacerlo todo. Las personas necesitan sentirse útiles, formarse y realizarse a través de su propio trabajo". $P_{5}$ : sentirse integrado en la empresa aumentará la motivación de los $\mathrm{RH}$.

\section{DISCUSIÓN}

No existe una metodología óptima y válida para todas las empresas, las decisiones que tomen los directores y líderes deben partir de un profundo conocimiento de las características y necesidades de todos los $\mathrm{RH}$ en la organización. Se debe de abandonar la forma tradicional de gestión de RH en la que los miembros de la organización eran vistos como un soporte del engranaje empresarial. Actualmente los $\mathrm{RH}$ son un factor clave en las empresas y de principal relevancia para la obtención de ventaja competitiva sostenible. El diseño de la gestión de RH debe enfocarse a mejorar el desempeño de los trabajadores y promover la unión de los objetivos de desarrollo personal con los de desarrollo de la organización.

El presente trabajo tras cinco años de interacción directa e informal con $\mathrm{RH}$ de diferentes niveles organizativos y diferentes departamentos ha permitido identificar una serie de factores de éxito en la gestión de la motivación de los $\mathrm{RH}$ de las empresas. Además, se profundiza en la gestión de estos para con los resultados dar respuesta a la pregunta de investigación ¿por qué empresas que invierten tiempo y recursos en gestión de $\mathrm{RH}$, llegando incluso a obtener certificaciones por ello, y cuando se pregunta a sus empleados están desmotivados e incluso muchos están buscando otras empresas para trabajar? 
Con los resultados del trabajo se han obtenido dos tipos de contribuciones a la literatura. En primer lugar, desarrollar un marco teórico integrado que permite estudiar cómo gestionar la motivación de los RH. Autores tradicionalmente investigaban factores por separado y este trabajo contribuye a la integración en una sola investigación de todos ellos. En segundo lugar, a través de un caso de estudio, se genera una hoja de ruta aplicable a directores y líderes en función del área funcional al que pertenezcan para que sepan qué acciones son determinantes para la gestión exitosa de cada factor. En la Tabla 1 se resumen las proposiciones planteadas en el modelo (una proposición por cada factor de éxito), las prácticas de $\mathrm{RH}$ realizadas por la organización y los resultados de gestión y comportamiento de $\mathrm{RH}$ conseguidos. Basado en el modelo teórico presentado en la Figura 3, se presenta evidencia obtenida en el caso de estudio de cada proposición formulada en base a las acciones realizadas y resultados obtenidos por la empresa objeto del caso de estudio.

\section{DISCUSION FINAL}

Respecto a la P1, la cultura organizativa debe ser una herramienta para diferenciarnos de competidores y atraer a los $\mathrm{RH}$ valiosos del mercado ya que serán pieza clave para el futuro de la organización. Para ello internamente debemos conseguir unidad entre equipos y departamentos. Es clave el papel jugado por los líderes, deben tener claro quienes son los $\mathrm{RH}$ valiosos más valorados y utilizarlos como ejemplo y referencia.

En la gestión de la P2, se debe trabajar en interactuar con los $\mathrm{RH}$ para conocer sus necesidades y realizar acciones que les hagan sentir orgullo de pertenencia. Es muy duro trabajar en empresas o departamentos que están compuestas por $\mathrm{RH}$ pesimistas, apáticos o que difunden información negativa. Se debe detectar a estos $\mathrm{RH}$ y acabar con su estancia en la organización de inmediato. Muchas veces no se despide a estos por el dinero que cuesta un acuerdo o indemnización, pero el coste que genera su presencia es mayor que el acuerdo de despido.

Dentro de la P3, se debe conocer que tipo de incentivo motiva a cada $\mathrm{RH}$ en cada etapa de su carrera profesional. Una mala gestión de la política de incentivos, en especial la selección y promoción de RH conlleva uno de los principales problemas encontrados en las empresas actualmente la pérdida de $\mathrm{RH}$ valiosos. El impacto de estos abandonos voluntarios suele ser doble. Por un lado, se pierde a una persona eficiente y que cumple objetivo para la empresa. Y, por otro lado, generalmente estos $\mathrm{RH}$ dada sus capacidades, encuentran trabajo en una empresa competidora y obtendrán buenos resultados para ella.

La P4, es uno de los factores más demandados en el entorno laboral actual. Si los RH no están satisfechos con su trabajo tendrán tensión y empeorara la relación con sus compañeros y como consecuencia la motivación. Y más grave, aparece el absentismo y abandono laboral. Para solventar este problema, no podemos obviar a la familia y amigos. Organizar eventos donde se invite a los seres cercanos hace que se incremente la unión e implicación de los RH con la empresa. Un trabajador satisfecho es mucho más productivo y para ello se deben de cuidar una serie de factores entre los que cabe destacar que el trabajo sea mentalmente desafiante, que las condiciones de trabajo sean las óptimas y que las decisiones de los directores sean justas.

Por último, para el éxito de la P5 el inicio de un RH en la organización a través del periodo de socialización se debe trabajar en su integración y adaptación. Esa imagen y sensaciones de las primeras horas o días muchas veces es imborrable. Debemos sitiar a RH valiosos, positivos y felices a liderar el proceso de socialización. Una vez integrados en la organización, los líderes deben de fomentar la existencia de relaciones sociales positivas, incentivar el trabajo en equipo y confiar en sus subordinados, de esta forma los trabajadores sentirán que la empresa es una prolongación de su hogar. Delegar es una de las tareas más complejas a la que se enfrentan directivos y líderes. Se debe formar para que su mentalidad tenga claro que si su carrera evoluciona van a depender de la confianza en su equipo ya que ellos no podrán abarcar todo el trabajo por realizar. En la Tabla 3 se presenta una síntesis de las proposiciones del estudio, las prácticas de RH implantadas por la empresa y los resultados más relevantes de gestión y comportamiento obtenidos.

En la literatura existente la mayoría de los trabajos se centran en el estudio de factores de motivación en el entorno laboral por separado, por ello la necesidad de ofrecer un modelo integrado de gestión con los factores más determinantes para el éxito y cómo se interrelacionan unos con otros. La motivación de los empleados no se consigue solo con uno de los factores, sino que se necesita de una confluencia de todos para poder generar una situación tan compleja de obtener como es la motivación.

La motivación de los trabajadores se trata de una temática que cada vez está teniendo mayor relevancia en el contexto empresarial, los directores y líderes se están cerciorando de su importancia a la hora de obtener ventajas competitivas sostenibles. Si se tienen bien definidos los pasos para ir creciendo dentro de la empresa y se da la posibilidad de hacerlo a los propios empleados frente a los trabajadores externos, este será un elemento que tendrá repercusión en la motivación de los $\mathrm{RH}$. Además, aportará retos y afán de superación a los $\mathrm{RH}$ de la organización con el consiguiente aumento de la eficiencia. 
Tabla 3. Proposiciones, prácticas y resultados sobre la gestión de RH y motivación

\begin{tabular}{|c|c|c|}
\hline \multicolumn{3}{|c|}{$\begin{array}{l}\text { ¿Por qué empresas invierten tiempo y recursos en gestión de } R H \text { y sus empleados están } \\
\text { desmotivados? }\end{array}$} \\
\hline Proposiciones & Prácticas de $R H$ & $\begin{array}{l}\text { Resultados de gestión y } \\
\text { comportamiento }\end{array}$ \\
\hline$P_{1}$ : Cultura & $\begin{array}{l}\text { Comunicación descendente } \\
\text { Valores informales } \\
\text { Características líderes }\end{array}$ & $\begin{array}{l}\text { Unión de subculturas } \\
\text { Atraer } \mathrm{RH} \text { valiosos } \\
\text { Diferenciación }\end{array}$ \\
\hline $\mathrm{P}_{2}:$ Clima & $\begin{array}{l}\text { Comparar empresas sector } \\
\text { Evaluación del desempeño } \\
\text { Reconocimiento público }\end{array}$ & $\begin{array}{l}\text { Orgullo de pertenencia } \\
\text { Conocer necesidades } \\
\text { Imitación } \mathrm{RH} \text { valiosos }\end{array}$ \\
\hline$P_{3}$ : Incentivos & $\begin{array}{l}\text { Bonos por objetivos } \\
\text { Desarrollo de carrera } \\
\text { Selección y promoción }\end{array}$ & $\begin{array}{l}\text { Etapas profesionales } \\
\text { Equidad y justicia } \\
\text { Retención } \mathrm{RH} \text { valiosos }\end{array}$ \\
\hline$P_{4}$ : Satisfacción & $\begin{array}{l}\text { Evolución continua } \\
\text { Apoyo de RH referentes } \\
\text { Conciliación laboral }\end{array}$ & $\begin{array}{l}\text { Polivalencia y autoeficiencia } \\
\text { Certidumbre y permanencia } \\
\text { Familia y amigos }\end{array}$ \\
\hline P5: Integración & $\begin{array}{l}\text { Periodo de socialización } \\
\text { Comunicación transversal } \\
\text { Dirección y liderazgo }\end{array}$ & $\begin{array}{l}\text { Realimentación positiva } \\
\text { Adaptación eficiente } \\
\text { Capacidad de delegación }\end{array}$ \\
\hline
\end{tabular}

\section{CONCLUSIONES}

De acuerdo con el trabajo presentado, a los resultados obtenidos, y la discusión planteada, se pueden plantear las siguientes conclusiones principales:

1.- Los líderes deben tener habilidades comunicativas, ser proactivos y ser flexibles. Así, la empresa conseguir atraer y retener a $\mathrm{RH}$ valiosos y suavizar las diferencias entre las diferentes subculturas empresariales que son claves para conseguir ventaja competitiva sostenible en el tiempo respecto a los competidores.

2.- El clima laboral es clave para conseguir que los $\mathrm{RH}$ sientan estén orgullosos de pertenecer a la empresa. Este orgullo llevará a los $\mathrm{RH}$ a implicarse en los objetivos y ser más eficientes. Se debe premiar y reconocer públicamente a los $\mathrm{RH}$ que destaquen en la consecución de los objetivos marcados para crear un deseo de imitación por el resto de los compañeros.

3.- La correcta gestión del desarrollo de carrera con ascensos justos y merecidos es más importante que los incentivos monetarios. En el contexto empresarial actual, los $\mathrm{RH}$ valiosos necesitan responsabilidad, autonomía y reconocimiento.

4.- Los RH este satisfechos de su empresa cuando evolucionan en su desarrollo de carrera, concilian su trabajo y vida personal y se sienten apoyados por sus superiores. Esta satisfacción generara una exitosa reputación, y los RH se implicarán en que sus familiares y amigos quieran entrar a formar parte de la organización.

5.- Los RH se sienten integrados cuando sus superiores los escuchan, y con la información obtenida se generan planes de acción coherentes. Cuando los líderes tienen la capacidad de confiar y delegar en sus equipos de trabajo se genera una situación de integración organizativa donde todos juntos trabajan para conseguir objetivos comunes.

\section{REFERENCIAS}

Adams, J.S., Toward an Understanding of Inequity, doi: 10.1037/h0040968, Journal of Abnormal and Social Psychology, 67(5), 422-436 (1963).

Álvarez-López, J., Apoyo de la Contabilidad de Dirección Estratégica a la Fidelización de la Clientela a través de la Motivación de los RH y de la Gestión de la Calidad Total, Revista de Dirección y Administración de Empresas, ISSN: 11352051, 2, 105-126 (1995).

Arévalo-Tomé, R, Caballero-Fernández G. y Quintás-Corredoira M., La Importancia de la Motivación en la Gestión de los $R H$, Revista Alta Dirección, ISSN: 0002-6549, 44(263), 9 (2009).

Bernal-Torres, C.A. y Blanco-Valbuena C.E., Innovación por Diseño y su Relación con las Variables del Entorno en una Muestra de Empresas en Bogotá-Colombia, doi: 10.4067/S0718-07642017000400017, Información Tecnológica, 28(4), $145-156$ (2017). 
Brown, S.P. y Peterson R.A., Antecedents and Consequences of SalesPerson Job Satisfaction: Meta-Analysis and Assessment of Casual Effects, doi: 10.2307/3172514, Journal of Marketing Research, 30, 63-77 (1993).

Cao, W. y Li Y., Research on Motivation System of Employees Analysis of Human Resources Management from a Psychological Perspective, doi: 10.1109/ICMSS.2010.5578035, International Conference on Management and Service Science, 15-19 (2010).

Carrillo, M, Padilla J., Rosero T. y Villagómez M.S., La Motivación y el Aprendizaje, Revista de Educación Alteridad, ISSN: 1390-325X, 4(2), 20-32 (2009).

Chiavenato, I., Administración de Recursos Humanos, McGraw Hill, Ciudad de México, México (2011).

Dadic, M., Bacic A. y Mihanovic I., The Role of Motivation in Human Resources Management, Interdisciplinary Management Research XIV, 14, 440-450 (2018).

Grant, R.M., The Resource-Based Theory of Competitive Advantage: Implications for Strategy Formulation, California Management Review, ISNN: 0008-1256, 33(3), 114-135 (1991).

Guerra, D. y Sansevero I., Principios y Competencia del Líder Transformacional en Instituciones Educativas, Revista de Educación Laurus, ISSN: 1315-883X, 14(27), 330-357 (2008).

Herzberg, F., Work and the Nature of Man, Word publishing, New York, United States (1966).

Jiang, K, Lepak D.P. y otros 4 autores, Clarifying the Construct of Human Resource Systems: Relating Human Resource Management to Employee Performance, doi: 10.1016/j.hrmr.2011.11.005, Human Resource Management Review, 22(2), 73-85 (2012).

Kotter, J.R. y Heskett J.L., Corporate Culture and Performance, Free press, New York, United States (1992).

Lago, A.A. y Wilson M.C., Human Resource Systems and Sustained Competitive Advantage: a Competency-Based Perspective, doi: 10.2307/258742, Academy of Management Review, 19(4), 699-727 (1994).

Lahera-Sánchez, A., La Participación de los Trabajadores en la Calidad Total: Nuevos Dispositivos Disciplinarios de Organización del Trabajo, doi: 10.2307/40184585, Revista Española de Investigaciones Sociológicas, 106, 63-102 (2004).

Lee, H.W., Performance-Based Human Resources Management and Federal Employee's Motivation: Modering Roles of Goal Clarifying Intervention, Appraisal Fairness, and Feedback Satisfaction, doi: 10.1177/0734371X17721300, Review of Public Personnel Administration, 39(3), 323-348 (2019).

López-Mas, J., Motivación Laboral y Gestión de RH en la Teoría de Frederik Herzberg, Revista de Investigación UNMSN, ISSN: 1810-8490, 8(15), 25-36 (2005).

Manterola, C., Revisión Sistemática de la Literatura: Síntesis de la Evidencia, Revista Medicina Clínica Condes, ISSN: 0716-8640, 20(6), 897-903 (2009).

Maslow, A.H., Motivación y Personalidad, Sagitario, Barcelona, España (1954).

Marañón-Rodríguez, E; Bauzá-Vázquez E. y Bello-Rodríguez A.A., La Comunicación Interna como Proceso Dinamizador para Fomentar Valores Institucionales y Sustento de una Identidad Universitaria, doi: 10.35362/rie4052488, Revista Iberoamericana de Educación, 40(5), 1-9 (2006).

Martini, E, Schadeck M., Wollenhaupt L. y Domingues A., La Motivación de los Sujetos en la Nueva Gestión de RH, UNR Journal, ISSN-e: 1852-0707, 2(14), 2160-2237 (2015).

McClelland, D.C., Toward a Theory of Motive Acquisition, doi: 10.1037/h0022225, American Psychologist, 20(5), 321-333 (1965).

Miles, J., Gónzalez A. y Mandirola N., Gestión de Alto Desempeño y su Impacto en los Resultados de la Empresa: el Caso de Uruguay y Argentina, doi: 10.4067/S0718-27242018000200057, Journal of Technology Management \& Innovation, 13(2) (2018).

Orlovic, L., Orlovic A. y Karlovic R., Working Motivation, Working Efficiency and Career Development as an Instrument of Human Resources Management - Perception of Police Officers, Policija I Sigurnost - Police and Security, ISSN: 13300229, 4(3), 226-246 (2015).

Ortiz, E.S., Lobos G. y Guevara D., Factores Determinantes del Bienestar Financiero y su Relación con la Calidad de Vida en una Muestra de Profesionales de Guayaquil, Ecuador, doi: 10.4067/S0718-07642019000100121, Información Tecnológica, 30(1) (2019).

Palma-Carrillo, S., Motivación y Clima Laboral, doi: 10.15381/rinvp.v3i1.4909, Revista de Investigación en Psicología, 3(1), 11-21 (2000).

Perry, C., Processes of a Case de Study Methodology for Postgraduate Research in Marketing, doi: 10.1108/03090569810232237, European Journal of Marketing, 32, 785-802 (1998).

Rajiani, I., Musa H. y Hardjono B., Ability, Motivation and Opportunity as Determinants of Green Human Resources Management Innovation, doi: 10.3923/rjbm.2016.51.57, Research Journal of Business Management, 37(5), 746-763 (2016).

Robbins, S.P., Comportamiento Organizacional, Pearson Prentice Hall, São Paulo, Brasil (2010). 
Rodríguez-Sánchez, J.L., Mora-Valentín, E.M. y Ortiz-de-Urbina-Criado, M., Successful Human Resources Management Factors in International Mergers and Acquisitions, doi: 10.3990/admsci8030045, Administrative Sciences, 8(3), 1-15 (2018).

Sandoval-Caraveo, M.C., Concepto y Dimensiones del Clima Organizacional, Hitos de Ciencias Económico Administrativas, ISSN: 2448-4733, 10(27), 83-87 (2004).

Salazar-Estrada, J.G, Guerrero-Pupo J.C., Machado-Rodríguez Y.B. y Cañedo-Andalia R., Clima y Cultura Organizacional: Dos Componentes Esenciales en la Productividad Laboral, Revista Cubana de Información en Ciencias de la Salud, ISSN: 2307-2113, 20(4), 67-75 (2009).

Skinner, B.F., Beyond Freedom and Dignity, Alfred A. Knopf, New York, United States (1971).

Tay, L.C., Tan F.Y. y Yahya K.K., The Power of Ability-Motivation-Oportunity Enhancing Human Resources Management Practices on Organizational Ethical Climate, International Journal of Business and Society, ISSN: 1511-6670, 18(3), 547562 (2017).

Villareal-Larrinaga, O. y Landeta-Rodríguez J., El Estudio de Casos como Metodología de Investigación Científica en Dirección y Economía de la Empresa. Una aplicación a la internacionalización, doi: 10.1016/S1135-2523(12)60033-1, Investigaciones Europeas de Dirección y Economía de la Empresa, 16, 1135-2523 (2010).

Vroom, V., Work and Motivation, Wiley, New York, United States (1964).

Yeung, A. y Berman B., Adding Value throug Human Resources, Human Resource Management, doi: 10.1002/(SICI)1099050X(199723)36:3<321::AID-HRM4>3.0.CO;2-Y, 36(3), 321-335 (1997).

Yin, R. K., The Abridged Version of Case Study Research. In Handbook of Applied Social Research Methods, (ed.) Leonard Thounsand Bickman and Debra J. Rog. Oaks: Sage Publications, 229-59, United States (1998). 